\title{
THE INFLUENCE OF WAKE CHOPPING ON WET-STEAM TURBINE MODELLING
}

\author{
Andrej Vasilj* \\ Hopkinson Laboratory \\ Engineering Department \\ University of Cambridge \\ CB2 $1 \mathrm{PZ}$ \\ UK \\ Email: av483@cam.ac.uk
}

\author{
Sebastian Schuster \\ Hopkinson Laboratory \\ Engineering Department \\ University of Cambridge \\ CB2 1PZ \\ UK
}

\author{
Alexander White \\ Hopkinson Laboratory \\ Engineering Department \\ University of Cambridge \\ CB2 1PZ \\ UK \\ Email: ajw36@cam.ac.uk
}

\section{ABSTRACT}

The formation of water droplets within condensing steam turbines is a complex process that occurs at supersaturated, nonequilibrium conditions and is influenced by the unsteady segmentation of blade wakes by successive blade rows. This is often referred to as 'wake chopping', and its effect on the condensation process is the subject of this paper. The practical significance is that thermodynamic 'wetness losses' (which constitute a major fraction of the overall loss) are strongly affected by droplet size. Likewise, droplet deposition and the various ensuing two-phase phenomena (such as film migration and coarse-water formation) also depend on the spectrum of droplet sizes in the primary fog.

The majority of wake-chopping models presented in the literature adopt a stochastic approach, whereby large numbers of fluid particles are tracked through (some representation of) the turbine flowfield, assigning a random number at each successive blade row to represent the particle's pitchwise location, and hence its level of dissipation. This study contributes to the existing literature by adding: (a) a comprehensive study of the sensitivity to key model parameters (e.g., blade wake shape and wake decay rate); (b) an assessment of the impact of circumferential pressure variations; (c) a study of the implications for wetness losses and $(d)$ a study of the implications for deposition rates.

\footnotetext{
*Address all correspondence to this author.
}

\section{NOMENCLATURE}

$d$

$G$

$h$

$h_{l g}$

$J$

$k$

$m_{l}$

$\mathrm{Kn}$

$\operatorname{Pr}$

$p$

$\dot{p}$

$q_{c}$

$r$

$s$

$T$

$T_{d}$

$\Delta T$

$t$

$\Delta t$

$\Delta t_{l s}$

V

W
Droplet diameter, [m]

Droplet growth rate, $\left[\mathrm{ms}^{-1}\right]$

Specific enthalpy, $\left[\mathrm{Jkg}^{-1}\right.$ ]

Specific enthalpy of vaporisation, $\left[\mathrm{Jkg}^{-1}\right]$

Nucleation rate, $\left[\mathrm{s}^{-1} \mathrm{~kg}^{-1}\right]$

Boltzmann's constant

Droplet mass, $[\mathrm{kg}]$

Knudsen number

Prandtl number

Pressure, $\left[\mathrm{Nm}^{-2}\right]$

Expansion rate

Condensation coefficient

Droplet radius, [m]

Specific entropy, $\left[\mathrm{Jkg}^{-1} \mathrm{~K}^{-1}\right.$ ]

Temperature, $[\mathrm{K}]$

Droplet temperature, $[\mathrm{K}]$

Subcooling, [K]

Time, [s]

Time increment, [s]

Time spent in the last stage stator, [s]

Velocity vector, $\left[\mathrm{ms}^{-1}\right]$

Relative velocity vector, $\left[\mathrm{ms}^{-1}\right]$

Wetness fraction

Efficiency

Thermal conductivity of steam, $\left[\mathrm{Wm}^{-1} \mathrm{~K}^{-1}\right]$

Density, $\left[\mathrm{kgm}^{-3}\right]$ 


$\begin{array}{ll}\sigma & \text { Surface tension, }\left[\mathrm{Jm}^{-2}\right] \\ \tau & \text { Wake decay time, }[\mathrm{s}] \\ \tau_{p} & \text { Particle relaxation time, }[\mathrm{s}] \\ \phi & \text { Kantrowitz correction factor } \\ \psi & \text { Mass flow function } \\ \Omega & \text { Rotational speed, }\left[\mathrm{s}^{-1}\right]\end{array}$

$\begin{array}{ll}\text { Subscripts } & \\ * & \text { Critical value } \\ l & \text { Liquid phase } \\ l s & \text { Last stage } \\ g & \text { Gas phase } \\ m & \text { Mixture } \\ r & \text { Radial direction } \\ s & \text { Saturation value } \\ 32 & \text { Sauter mean value } \\ \theta & \text { Circumferential direction } \\ \infty & \text { Average value }\end{array}$

\section{INTRODUCTION}

Condensing steam flow in turbines is a topic that has been investigated for decades and has a significant influence on turbine performance, yet a large number of questions remains unanswered. As the superheated steam crosses the saturation line, it does not condense immediately but enters a supersaturated, subcooled, non-equilibrium state. At the location of maximum subcooling, the Wilson point, it will revert to equilibrium through the condensation process: latent heat released by the phase change process will reduce the subcooling, bringing the steam back to equilibrium. Nucleated droplets will continue to grow as the flow passes through the machine. Both nucleation theory and droplet growth have been extensively researched by a large number of authors (see the reviews by McDonald (1962, 1963), Young (1982), Bakhtar et al. (2005)) and the models have shown excellent predictions of wetness formed in nozzle expansion experiments (e.g., Bakhtar et al. (1976), White (1992)). The droplet spectrum formed by a nozzle expansion is narrow, monodispersed and has a low mean droplet diameter, as shown in Fig. 1. However, optical probe measurements in turbines (Walters (1985), Young et al. (1988), Skillings et al. (1989)) have shown polydispersion of the droplet spectrum and a notably larger mean diameter, thus pointing to a discrepancy which could not be explained using the established methods. A number of authors (Bakhtar \& Heaton (2005), Petr \& Kolovratnik (2000), Guha \& Young (1994)) suggest that the discrepancy originates from the two-dimensional blade unsteadiness in the form of wake chopping.

The concept of wake chopping is associated with rotational motion of turbine blades, as first described by Gyarmathy \& Spengler (1974). Considering a single stream surface, parcels of superheated steam enter a blade row at different pitchwise lo-

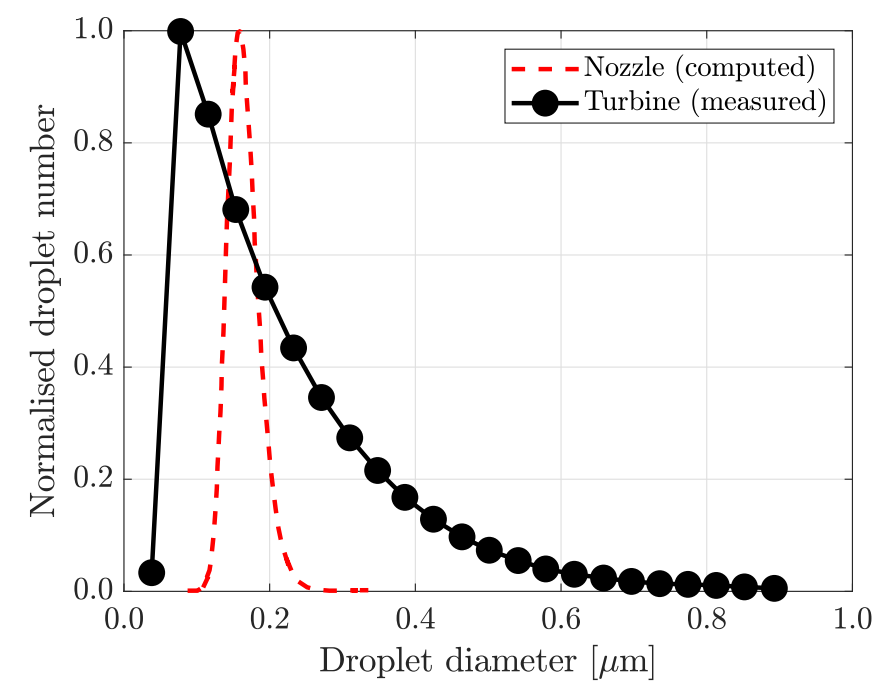

FIGURE 1. Droplet size spectrum comparison between nozzles and turbines, reproduced from Young (1992).

cations. The ones that enter in the core of the flow expand almost isentropically, while those close to the blades experience significant viscous losses, forming blade wakes. Consequently, parcels in wake regions have a higher temperature and lower velocity upon leaving the blade row than their core-flow counterparts. The wakes are segmented in a periodic fashion upon entering the next blade row due to relative motion between rotors and stators, causing fluctuations of flow properties in subsequent blade rows.

The foundations of wake chopping models were presented by Gyarmathy \& Spengler (1974), and provide a methodology for the analysis of the steam temperature fluctuations observed by Wood (1973). Their ideas were subsequently implemented as a proof of concept by Bakhtar \& Heaton (1989) and further developed with a more theoretical basis by Guha \& Young (1994). The stochastic modelling approach to wake chopping is based on an efficiency distribution across the pitch and the random pitchwise inlet location of fluid parcels at the leading edge of blade rows, thus effectively defining a set of random thermodynamic histories through the machine. The variable levels of loss each parcel experiences due to individual flow histories lead to the spread of the nucleation zone over a range of expansion rates, thus broadening the droplet size spectrum.

The current body of literature shows that the wake modelling capabilities are important, especially in the context of fast onedimensional and two-dimensional calculation procedures. However, the sensitivity of wake chopping to parameter variation (wake profile, wake decay rate, pitchwise pressure), parameter interaction, and its impact on the droplet size spectrum is still not fully understood. Even though these modelling methods have already been applied and investigated in the context of Walters' measurements, current paper not only expands on the initial find- 
ings by Guha \& Young (1994) and Petr \& Kolovratnik (2000) but also provides a unique insight into how wake chopping influences thermodynamic relaxation loss while also defining the design context in which wake chopping is of high importance.

\section{THE COMPUTATIONAL MODEL}

Expansion of superheated steam causes its condition to cross the saturation line and enter a meta-stable supersaturated state. With further expansion, the flow tends to revert back to equilibrium through the latent heat release of the phase change process in which a large number of water droplets is formed.

Non-equilibrium steam condensation is modelled using classical homogeneous nucleation theory, as discussed by Bakhtar et al. (2005).

$$
J=\frac{1}{1+\phi} q_{c} \frac{\rho_{g}^{2}}{\rho_{l}} \sqrt{\frac{2 \sigma}{\pi m^{3}}} \exp \left(\frac{-4 r_{*}^{2} \pi \sigma}{3 k T_{g}}\right)
$$

The symbols in this expression are standard, and are defined in the notation. $(1+\phi)$ is Kantrowitz's non-isothermal correction, details of which are given, with discussion, in Kantrowitz (1951) and Bakhtar et al. (2005). Growth of nucleated droplets, determined using Young (1982) droplet growth model, is calculated as:

$$
G=\frac{\mathrm{d} r}{\mathrm{~d} t}=\frac{\lambda\left(T_{s}-T_{g}\right)\left(1-\frac{r_{*}}{r}\right)}{h_{l g} \rho_{f} r\left[1+3.78(1-v) \frac{\mathrm{Kn}}{\mathrm{Pr}}\right]}
$$

where the term $\left(1-r_{*} / r\right)$ improves the accuracy of the droplet growth model for droplet sizes near the critical radius $r_{*}$. Furthermore, $v$ is a function proposed by Young that improves agreement with low pressure nozzle experiments and its full definition, as well as the justification for its use, can be found in Young (1982) and White \& Young (1993). Steam properties are determined using the IAPWS-IF97 (1997) formulation in the shape of table look-up outlined by Hill et al. (2000).

Droplets are considered to be uniformly distributed and mixture properties are calculated through the use of wetness fraction y. E.g., for enthalpy:

$$
h=(1-y) h_{g}\left(T_{g}, p\right)+y h_{l}\left(T_{s}, p\right)
$$

While the steam enthalpy is evaluated at local pressure and steam temperature, droplet enthalpy is calculated at saturation temperature and local pressure, thus neglecting the variation in water properties with the change in droplet diameter due to capillarity effects. However, this is a reasonable estimate since the effect is substantial only for very small diameters.

\section{Wake chopping model}

The stochastic model of wake chopping implemented here is similar to that developed by Guha \& Young (1994). It is based on independent calculations for each streamsurface placed at intervals from hub to shroud, while neglecting radial mixing between the streamsurfaces and radial wake deformations. All calculations presented in this paper are performed for a mid-span streamsurface.

The calculations are performed by tracking a large number of steam parcels in a Lagrangian frame of reference as they expand through the turbine flowfield using the wet-steam routines developed by Young (1992). The wake chopping effect is modelled in a statistical sense by assigning a random dimensionless mass flow function $\psi$ to individual parcels upon entering each blade row. Pitchwise entry location for every parcel is considered to be completely independent of its history, thus performing a random walk through the turbine and capturing the nature of wake chopping. A polytropic efficiency profile $(\eta)$ as a function of a dimensionless pitchwise mass flow function is associated with each blade row, as shown in Fig. 2. Thus, parcels passing through the core of the flow expand almost isentropicaly $(\psi \rightarrow \pm 0.5)$, while those passing through the wake region will experience a higher loss due to a progressive efficiency decrease with the proximity to the wall (blade surface, $\psi \rightarrow 0$ ). The efficiency profiles could be determined from measurements or from Computational Fluid Dynamics (CFD) calculations, but one of the objectives here is to determine the sensitivity of results to the shape of these profiles. The wake shapes are thus artificially defined using simple mathematical representations (linear, parabolic, square, gaussian), while keeping the mean value of polytropic efficiency and the standard deviation of the resulting fluctuations constant. Nucleation rate, droplet growth and thermodynamic relaxation loss are predicted by marching downstream, based on the determined axial pressure and efficiency distribution for every steam parcel. The local time step is determined based on local gradients (expansion rate, nucleation rate and droplet relaxation time) and a new droplet size group is generated for each nucleating time step. Having defined the efficiency and pressure decrease for the current streamsurface, mixture enthalpy change is defined through the Lagrangian form of the energy equation. However, individual pathlines on the same streamsurface will interact with each other via turbulent mixing and heat conduction processes. This is modelled with a diffusion-like term, as described by Guha \& Young (1994), leading to the following form of the energy equation:

$$
\frac{\mathrm{D} h}{\mathrm{D} t}-\frac{1}{\rho} \frac{\mathrm{D} p}{\mathrm{D} t}=-\frac{(1-\eta)}{\rho} \frac{\mathrm{D} p}{\mathrm{D} t}-\frac{\left(h-h_{\infty}\right)}{\tau}
$$

where $h_{\infty}$ is the mixture enthalpy value that would result if mixing between streamlines were instantaneous, and $\tau$ is the time 


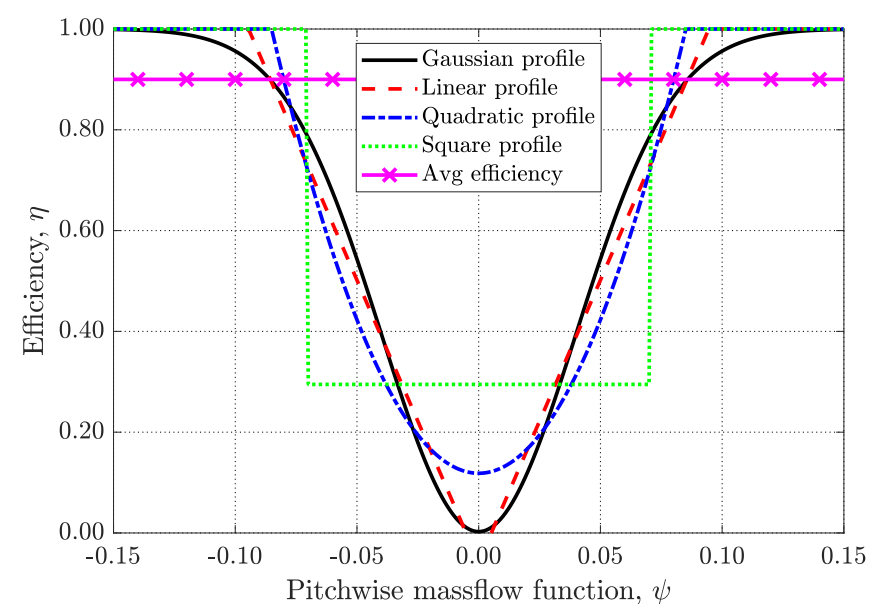

FIGURE 2. Artificial blade wake profiles.

constant associated with mixing out of gradients. Large values $(\tau \rightarrow \infty)$ correspond to negligible mixing while small values $(\tau \rightarrow 0)$ imply instantaneous equilibriation between streamlines. A simple analysis presented by Guha \& Young (1994) estimated wake rothalpy differences decrease at a rate of approximately $\tau=5 \Delta t_{l s}$, where $\Delta t_{l s}$ is wake transit time in the last turbine stage stator. This was adopted here as a reasonable estimate of the realistic turbulent diffusion in a wake for calculations with a fixed wake decay rate while varying other parameters. To fully describe the mixing process (i.e., the wake decay) it is necessary to specify $h_{\infty}$. It can be determined as the average value of a statistically meaningful sample of pathline calculations - however, to avoid doing a runtime correction of $h_{\infty}$, it is possible to estimate its value using the average efficiency $\eta_{\infty}$ :

$$
\frac{\mathrm{D} h_{\infty}}{\mathrm{D} t}-\frac{1}{\rho} \frac{\mathrm{D} p}{\mathrm{D} t}=-\frac{\left(1-\eta_{\infty}\right)}{\rho} \frac{\mathrm{D} p}{\mathrm{D} t}
$$

where $\mathrm{D} / \mathrm{D} t$ is the material derivative that can be expressed as: $\mathrm{D} / \mathrm{D} t=\partial / \partial t+(\mathbf{v} \cdot \nabla) . \quad h_{\infty}$ is thus determined using a single pathline calculation prior to the wake chopping modelling.

\section{Test case}

The majority of turbines have different blade geometries and, therefore, different expansion rates between blade rows, which change the flow conditions at the Wilson point depending on the blade row at which the nucleation occurs. The changes to the droplet size spectrum obtained on such a geometry are difficult to interpret since the wake chopping contribution cannot be decoupled from the influence of local geometrical characteristics. To tackle this issue, the sensitivity of droplet size spectra to wake chopping parameters was isolated using a repeating-stage turbine design and analysed in one dimension (meridional representation of the mid-streamsurface) using a representative axial pressure variation (pressure ratio of 0.6 per stage), along the passage centre and the blade pressure and suction surfaces.

Pressure distributions were extracted from CFD results by Hughes (2016), with Fig. 3 showing the axial variation of pressure along the passage centre. Pressure and suction surface pressure distributions were extracted from Hughes (2016) and scaled based on the blade loading (calculated from blade flow angles in a throughflow calculation). This allows for cross-passage (pitchwise) variations of expansion rate to be taken into account in the wake chopping model. A simple linear variation of pressure is assumed between suction and pressure surfaces.

The test case was designed with eight stages in order to increase the possible number of pathline histories and prolong the remaining time for nucleated droplets to grow. This makes the effect of wake chopping parameter perturbations on droplet size spectrum clearer and easier to interpret.

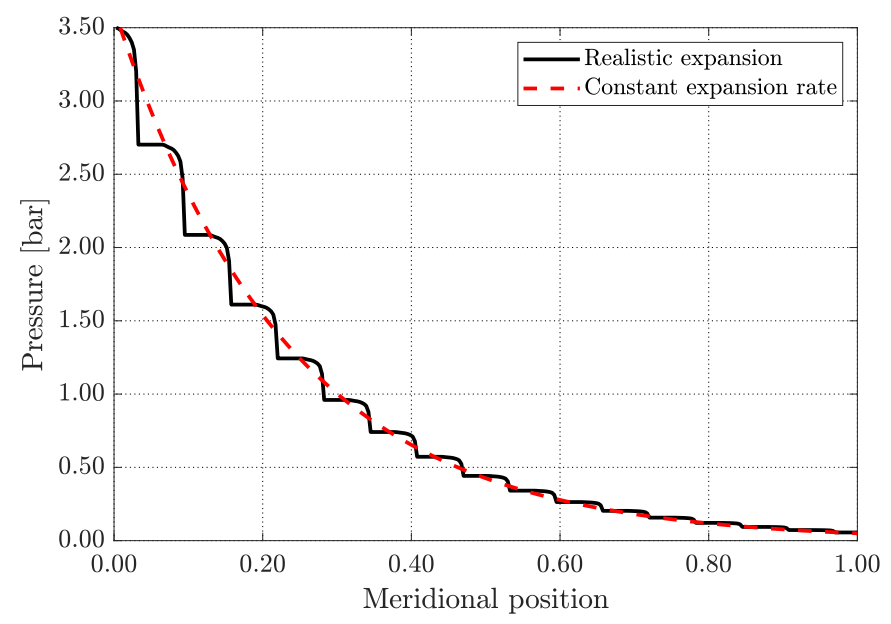

FIGURE 3. Axial pressure distribution for a constant and a variable expansion rate.

\section{Data management}

In order to reach the desired convergence criteria (runtime efficiency average is $\pm 0.5 \%$ from $\eta_{\infty}$ ), tracking between 5000 and 10000 parcels was required, with every condensing pathline resulting in a discrete number of droplet size groups $(\sim 200$ per pathline). Since every pathline has a unique path through the machine, the calculations result in a considerable amount of data. The stochastic approach can be efficiently programmed by storing information on the current pathline and an 'aggregate pathline', to which the results from each pathline are added, thus significantly reducing memory requirements. Due to the large number of droplet size groups aggregated throughout the calculation, individual droplet groups are split into a number of droplet size 
bins and presented in terms of mass fraction of liquid per mass of mixture $\left(\mathrm{kg}_{l} / \mathrm{kg}_{m}\right)$ (normalised using the peak value), thus significantly reducing the size of the final dataset.

\section{Droplet deposition model}

The impact of wake chopping on deposition is assessed by recording the droplet spectrum at the final rotor leading edge and performing a deposition calculation. Only inertial deposition is considered here, as an example of how wake-choppinginduced changes to the droplet spectra affect subsequent twophase processes. Droplets are considered to be spherical and non-interacting and further droplet growth is neglected. Inertial deposition is calculated by assuming viscous drag as the only force affecting the droplet motion. The equations are written in the cylindrical polar coordinate system rotating with the same angular velocity as the blades (i.e., zero for stators and $\Omega$ for rotors). Droplets are sufficiently small $(0.1 \leq d \leq 2 \mu \mathrm{m})$ so that radial centrifuging and axial drift may be neglected. Integrating the momentum equation in a Lagrangian framework following the method of Young \& Yau (1988) gives:

$$
\Delta W_{\theta 2}=\beta \Delta W_{\theta 1}+\tau_{p}(1-\beta)\left[\frac{\mathrm{D} W_{\theta g}}{\mathrm{D} t}+\frac{W_{r l} W_{\theta l}}{r}+2 \Omega W_{r l}\right]
$$

where 1 and 2 represent integration step start and end points respectively, $\Delta W_{\theta}=\left(W_{\theta g}-W_{\theta l}\right)$ is the circumferential relative velocity difference between steam and the droplets, and $\beta=\exp \left(-\Delta t / \tau_{p}\right)$.

The repeating-stage blade geometry was extracted from Hughes (2016) and pitchwise steam trajectories on the streamsurface were approximated by a linear combination of pressure and suction side curves. Since the goal is to demonstrate deposition rates in a model flowfield, steam velocity was determined from a coarse streamline curvature calculation. Droplets were assumed to enter the blade row with no velocity slip at the blade leading edge. When passing through a blade row, droplets deviate from the steam path and their drift value is assessed against blade surface coordinates to determine whether they are deposited.

\section{SENSITIVITY TO MODELLING ASSUMPTIONS}

The following selection of results, taken from a more comprehensive study (Vasilj (2020) [manuscript in preparation]), serves to illustrate the impact of wake chopping on the condensation process and to determine the sensitivity of results to the modelling assumptions and main modelling parameters.

\section{Wake shape}

The polytropic efficiency distribution defines the loss experienced by individual parcels passing through each blade row.
Coupled with a random path through the machine, it moves the position of the Wilson point to locations with a differing expansion rates, thus affecting the number of droplets nucleated and hence their subsequent size. In order to decouple the influence of different flow parameters (e.g., expansion rate and wake decay rate) that might obscure the effect of efficiency distribution on the generated droplet size spectrum, the calculations were initially performed using a constant expansion rate $\left(\dot{p}=\ln \left(p_{2} / p_{1}\right) / \Delta t\right.$, where 1 and 2 signify start and end point of the time step) and persisting wakes $(\tau \rightarrow \infty)$. The droplet size spectra at the outlet are shown in Fig. 4 for each of the four wake profiles.

Some differences in the detailed shape of the size distributions are observed which in this case come from different dissipation histories experienced by the fluid particles, expansion rates being the same in all cases. It should be noted, however, the differences are quite modest given the sizeable variations in wake shape, and are only significant for the discontinuous, square wake profile. Note that the wake widths have been scaled such that the standard deviation of fluctuations is the same for each profile. Moreover, the discrepancies are much less pronounced when wake mixing and variable expansion rate are taken into account (see section: Wake shape revisited).

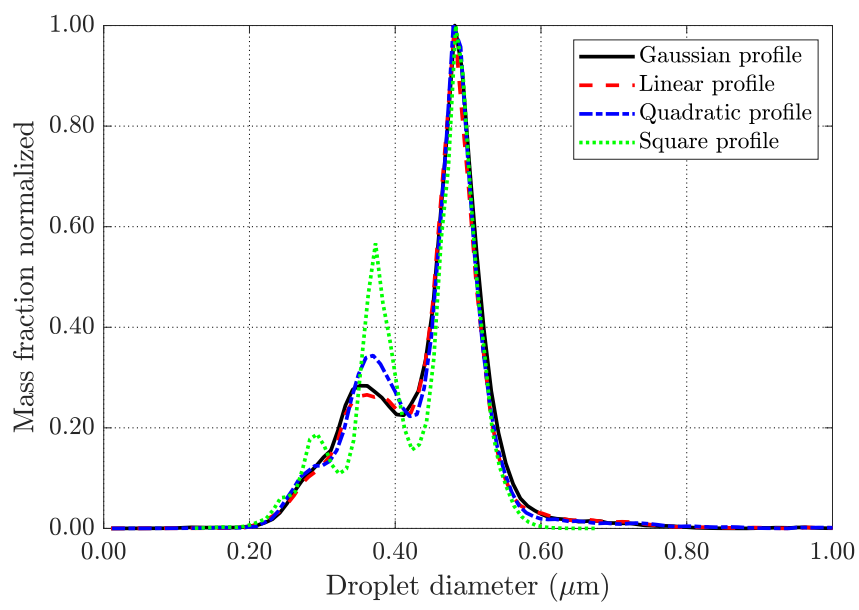

FIGURE 4. Wake shape influence assuming a fixed expansion rate and no wake decay $(\tau \rightarrow \infty)$.

\section{Wake decay rate}

Turbulent diffusion and thermal conduction in a blade passage play a major role in smoothing out the wake-induced fluctuations. The high entropy wakes are assumed to mix out with time constant $\tau$, as modelled by the last term in Eq. 4. $\tau$ can be estimated from simple physical modelling (see Guha \& Young (1994)) or from CFD, but cannot easily be determined with any 
precision. A range of values for $\tau$ were tested on $1 \mathrm{D}$ and $2 \mathrm{D}$ variable axial expansion rate cases. The value of $\tau$ might reasonably be estimated to within a factor of $5\left(2<\tau / \Delta t_{l s}<10\right)$. Figure 5 shows a significantly different droplet spectrum with $\tau / \Delta t_{l s}=10$, with a second peak at $d \approx 0.85 \mu \mathrm{m}$. However, the sensitivity to $\tau$ is much less pronounced when pitchwise pressure variations are taken into account, as shown in Fig. 6 and discussed further below.

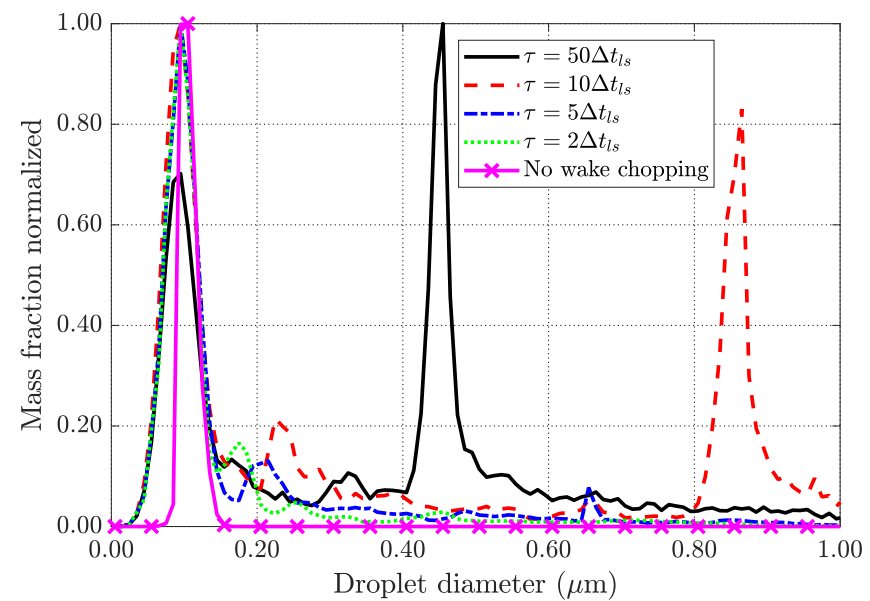

FIGURE 5. Droplet spectrum sensitivity to wake decay for a 1D, variable $\dot{p}$.

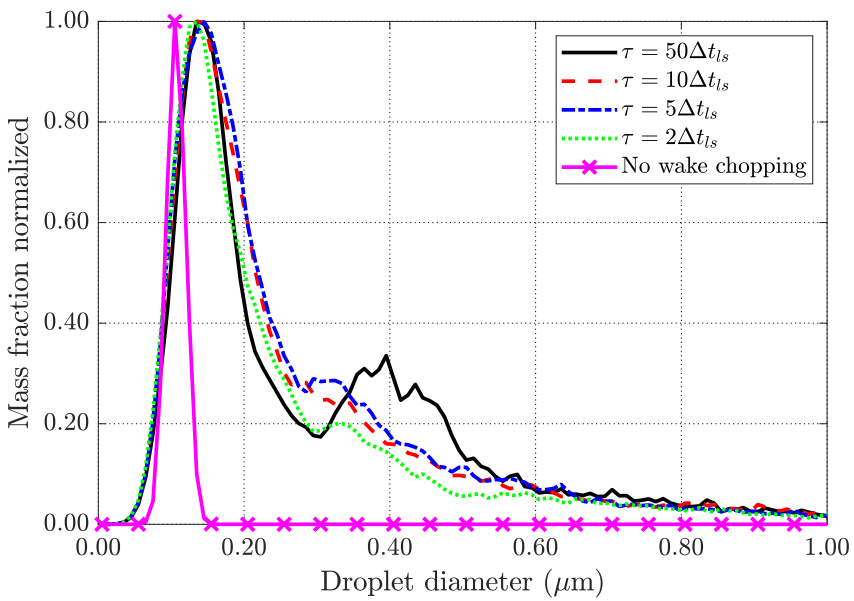

FIGURE 6. Droplet spectrum sensitivity to wake decay for a 2D, variable $\dot{p}$.

\section{Pitchwise pressure variation}

The water droplet formation process is strongly influenced by the local expansion rate, since high expansion rates lead to nucleation of a higher number of small droplets. Therefore, the diversity of expansion rates found in the nucleation zone has a strong impact on the generated droplet spectrum. Figure 7 shows the difference in the droplet size spectrum when taking the pitchwise pressure variation into consideration. The 1D pressure variation here corresponds to the mid-passage distribution shown previously in Fig. 3. The underlying spectrum shape for the $2 \mathrm{D}$ case is similar to that for $1 \mathrm{D}$, but the additional pitchwise variations increase the range of expansion rate in the nucleation zone, causing widening of the spectrum. By contrast, expansion with a constant $\dot{p}$ generates a narrow spectrum with a very different shape. In this case, wake chopping varies the axial location of nucleation (thereby changing the remaining time available for droplets to grow) but the final droplet size is determined chiefly by the number of droplets nucleated and, hence, by $\dot{p}$ in the Wilson zone.

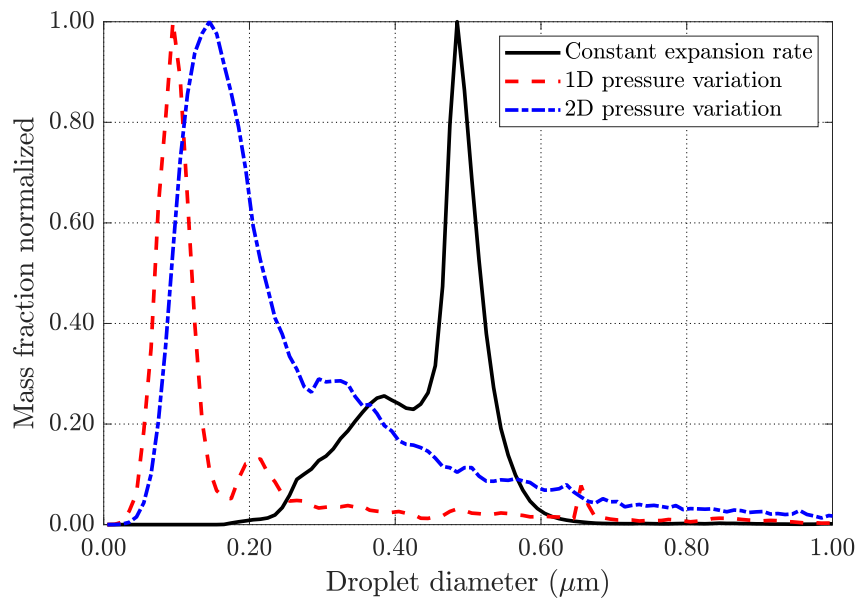

FIGURE 7. Impact of $1 \mathrm{D}$ and $2 \mathrm{D}$ pressure variation on droplet size spectrum for a fixed wake decay rate $\left(\tau=5 \Delta t_{l s}\right)$.

\section{Wake shape revisited}

The limited impact of wake shape variation on droplet size in case of a constant $\dot{p}$ is further diminished by accounting for wake mixing, and 2D expansion rate variation. Despite the difference in wake profile description, the droplet size spectra are very much alike, as seen in Fig. 8. Droplets with different thermodynamic histories, due to random pitchwise position in each blade row, will reach the Wilson point at a different axial locations, spreading of the nucleation zone. This will diffuse the effect of wake discontinuities (e.g., square wake) making the droplet spectrum prediction more robust. 


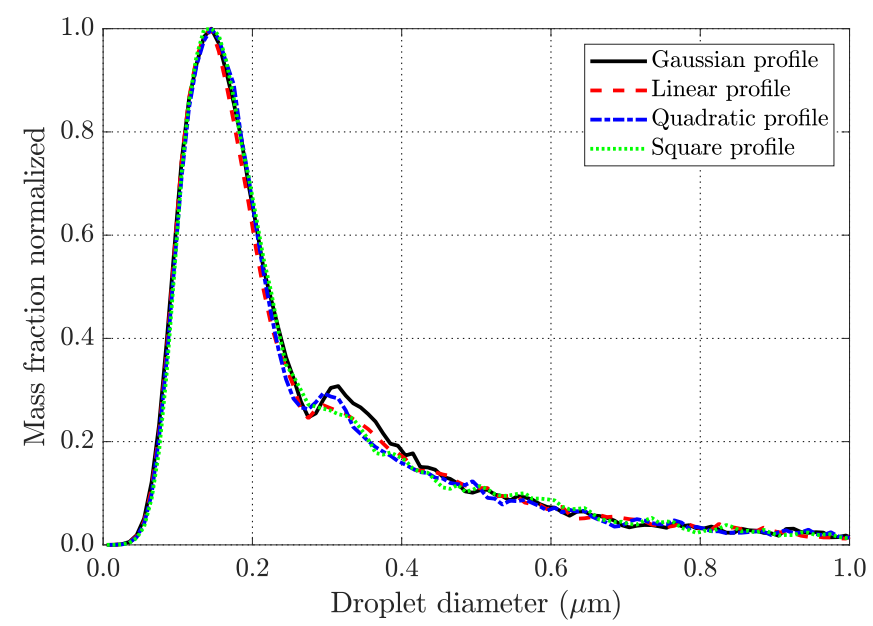

FIGURE 8. Wake shape influence assuming 2D variable expansion rate and wake decay $\left(\tau=5 \Delta t_{l s}\right)$.

\section{WAKE CHOPPING INFLUENCE ON TURBINE PERFOR- MANCE}

Wake chopping has a significant influence on the shape of the droplet size distributions as shown in the previous sections. The consequences of these changes are of interest in the context of turbine design. Figure 9 shows the difference in outlet wetness fraction depending on the included effects, and for a range of inlet superheat temperatures, which shift the axial location of nucleation. The impact of the different modelling assumptions on outlet wetness fraction is relatively small. This is to be expected because the equilibrium wetness is determined by the pressure and entropy. However, wake chopping has a more pronounced

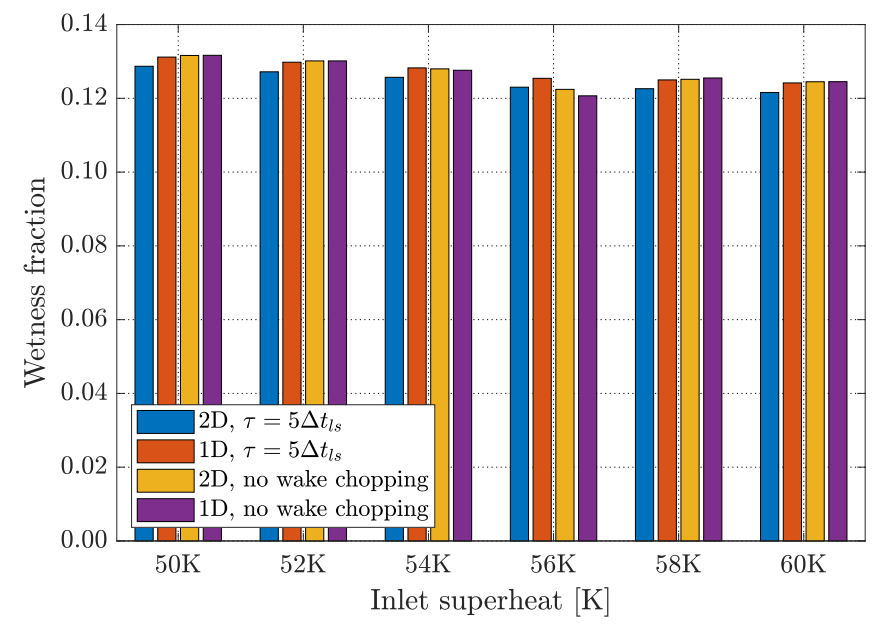

FIGURE 9. The impact of wake chopping on outlet wetness for a range of inlet superheat values. impact on the relaxation loss (defined as the component of entropy generation due to irreversible phase change) shown in Fig. 10. Note that the entropy changes have been normalised by the specific gas constant, making the values comparable to a fractional stagnation pressure loss. Axial pressure variation with no wake chopping causes each nucleation event to be confined to a singular expansion rate, generating a narrow droplet spectrum. This makes the thermodynamic relaxation loss sensitive to the axial location of the Wilson point and, thereby, dependent on the inlet temperature. By increasing the inlet temperature, nucleation moves from the blade passage to the inter-blade gap where pressure is almost constant - therefore $\dot{p} \approx 0$. This occurs for an inlet superheat of $56 \mathrm{~K}$ and leads to lower outlet wetness because the flow does not reach equilibrium. For a two-dimensional pressure field, however, the nucleation events are spread over a number of expansion rates at approximately the same axial location of the Wilson point, thus broadening the droplet size spectrum even in the absence of wake chopping. This makes the relaxation loss less sensitive to inlet temperature. Inclusion of wake chopping spreads the nucleation over a broad axial zone that may span two blade rows, hence further reducing the sensitivity to inlet superheat. Thus, as shown in Fig. 10, the relaxation loss for the 2D wake-chopping model is more or less independent of inlet superheat.

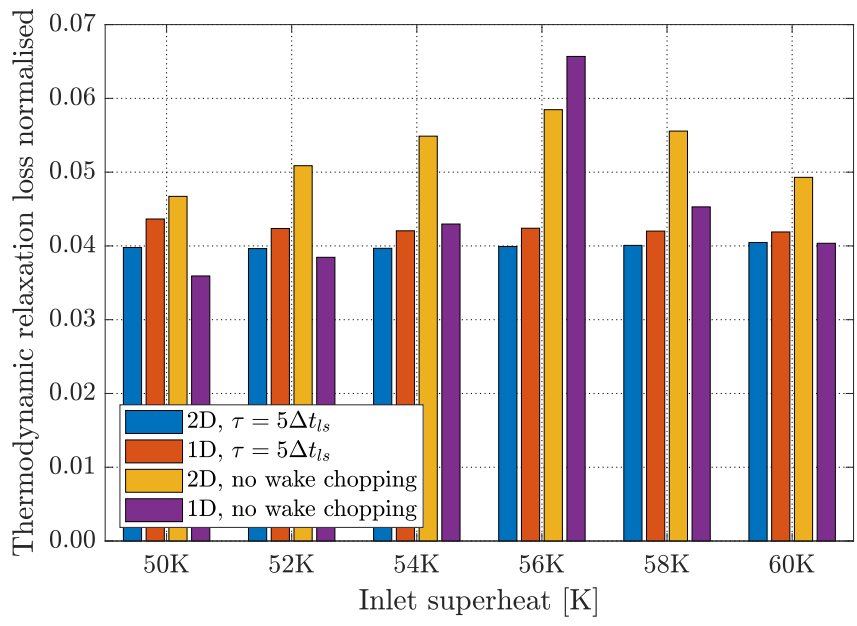

FIGURE 10. The impact of wake chopping on thermodynamic relaxation loss for a range of inlet superheat values.

\section{Deposition}

Depositional processes are strongly dependent on particle relaxation time; widening the droplet spectrum and increasing the average size leads to increased deposition rates. This can be seen in Fig. 11: function $\mathrm{d} m / \mathrm{d} r$ is presented for inertial deposi- 
tion with and without wake chopping (both curves are normalised with the peak value of no wake chopping deposition rate), where the wake chopping droplet distribution leads to a significantly larger percentage of deposited liquid mass $(\approx 3 \times$ higher deposition rate). Most of the droplets have a diameter of $\sim 0.1 \mu \mathrm{m}$ and a low particle relaxation time, hence closely following steam streamlines and causing only a small fraction of them to impinge on the surrounding solid surfaces. Yet, due to their high concentration in the droplet-steam mixture, this results in a large mass of liquid being deposited, as observed by the left peak in the distribution. Since particle relaxation time is proportional to droplet diameter, droplet groups with larger diameter develop larger velocity slip which leads to an increase in deposition rates. Even though the concentration of large droplets in wet steam flow is low, each deposited droplet contributes a large amount of liquid mass (which scales with $d^{3}$ ). Hence, any local extrema in the concentration of large droplets (which is the case with bimodal droplet size distributions) will be strongly reflected on the deposition curve and overall deposited liquid mass. The difference in deposition rates will affect liquid films, coarse water formation and all of the associated losses. Therefore, it is highly important for turbine design and performance to capture the changes in the droplet size spectrum shape due to wake chopping.

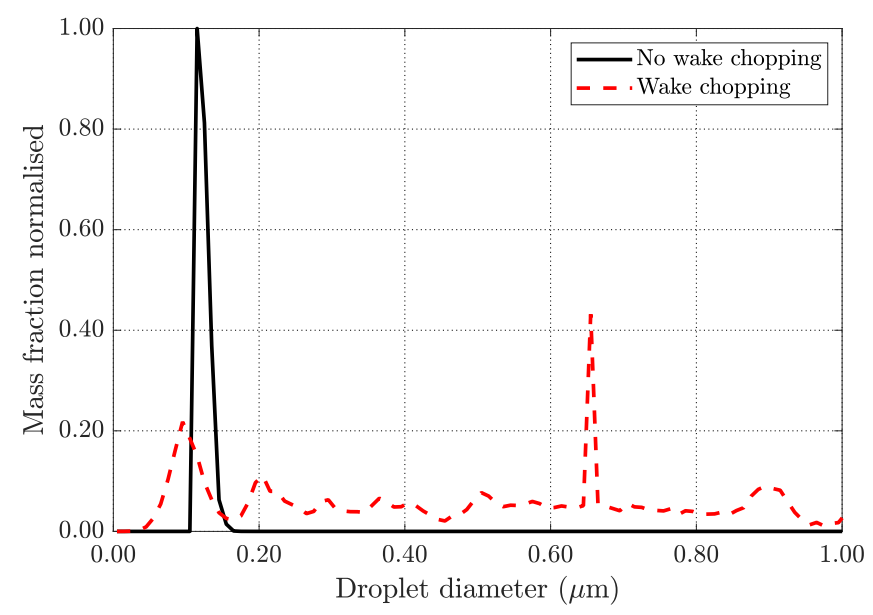

FIGURE 11. Inertial deposition with and without wake chopping.

\section{CONCLUSIONS}

A non-equilibrium wet-steam wake-chopping model has been presented, suitable for incorporation into streamline curvature or other throughflow calculation methods. The model follows a similar approach to previously published work by other authors, but includes a study of the sensitivity of results to the key modelling parameters. Pitchwise variations in pressure and (inertial) deposition are included, as well. The main conclusions are:

(i) The predicted droplet spectra are more or less insensitive to the wake shape, provided both mean loss and standard deviation - i.e., the effective wake width - are held constant. Previous studies show a dependence on the wake shape because the effective width was not held constant.

(ii) Inclusion of pitchwise pressure variation also renders the results less sensitive to other modelling parameters (notably the wake decay rate) by spreading nucleation over a broader range of expansion rates. Predicted droplet spectra and relaxation losses became less sensitive to inlet temperature for similar reasons.

(iii) In keeping with previous work and optical measurements, droplet spectra are predicted to be much broader as a result of wake chopping. This has a significant impact on the inertial deposition rate, and is likely to have an even more pronounced effect on turbulent deposition, which is known to vary strongly with Stokes number.

The conclusions of this study are directly applicable to wake chopping modelling on realistic turbine geometries since the broader range of expansion rates in the nucleation zone (due to different geometries between blade rows) will further reduce the sensitivity of wetness prediction, making results even less sensitive to modelling parameters.

\section{ACKNOWLEDGMENT}

The first author, AV, is grateful for the support of the Engineering and Physical Sciences Research Council (EPSRC). All authors also wish to acknowledge the generous support from General Electric.

\section{REFERENCES}

Bakhtar, F. \& Heaton, A. (1989), An examination of the effect of wake choppingon droplet sizes in steam turbines, in 'Technology of turbine plant operating with wet steam', Thomas Telford Publishing, pp. 197-200.

Bakhtar, F. \& Heaton, A. V. (2005), 'Effects of Wake Chopping on Droplet Sizes in Steam Turbines', Proceedings of the Institution of Mechanical Engineers, Part C: Journal of Mechanical Engineering Science 219(12), 1357-1367.

Bakhtar, F., Young, J. B. \& Ghoneim, Z. (1976), 'A study of nucleating and wet steam flows in turbines', Proceedings of the Institution of Mechanical Engineers 190(1), 545-559.

Bakhtar, F., Young, J. B., White, A. J. \& Simpson, D. A. (2005), 'Classical Nucleation Theory and Its Application to Condensing Steam Flow Calculations', Proceedings of the Institution of Mechanical Engineers, Part C: Journal of Mechanical Engineering Science 219(12), 1315-1333. 
Guha, A. \& Young, J. (1994), 'The Effect of Flow Unsteadiness on the Homogeneous Nucleation of Water Droplets in Steam Turbines', Philosophical Transactions of the Royal Society of London. Series A: Physical and Engineering Sciences 349(1691), 445.

Gyarmathy, G. \& Spengler, P. (1974), 'Flow fluctuations in multistage thermal turbomachines', Traupel commemorative volume.(A 75-19051 06-02) Zurich, Juris-Verlag AG, 1974, p. 95.

Hill, P., Miyagawa, K. \& Denton, J. (2000), 'Fast and accurate inclusion of steam properties in two-and three-dimensional steam turbine flow calculations', Proceedings of the Institution of Mechanical Engineers, Part C: Journal of Mechanical Engineering Science 214(7), 903-919.

Hughes, F. (2016), Throughflow methods for two-phase flow in steam turbines., $\mathrm{PhD}$ thesis, University of Cambridge.

IAPWS-IF97 (1997), 'Revised release on the IAPWS industrial formulation for thermodynamic properties of water and steam, internet resource'.

URL: http://www.iapws.org/release.html

Kantrowitz, A. (1951), 'Nucleation in Very Rapid Vapor Expansions', The Journal of Chemical Physics 19(9), 1097-1100.

McDonald, J. E. (1962), 'Homogeneous nucleation of vapor condensation. I. Thermodynamic aspects.', American Journal of Physics .

McDonald, J. E. (1963), 'Homogeneous nucleation of vapor condensation. II. Kinetic aspects', American Journal of Physics 31(1), 31-41.

Petr, V. \& Kolovratnik, M. (2000), 'Modelling of the droplet size distribution in a low-pressure steam turbine', Proceedings of the Institution of Mechanical Engineers, Part A: Journal of Power and Energy 214(2), 145-152.

Skillings, S. A., Moore, M. J., Walters, P. T. \& Jackson, R. (1989), A reconsideration of wetness loss in LP steam turbines, in 'Technology of turbine plant operating with wet steam', pp. 171-177.

Vasilj, A. (2020), Comprehensive throughflow method for steam turbines., PhD thesis, University of Cambridge.

Walters, P. (1985), Wetness and efficiency measurements in lp turbines with an optical probe as an aid to improving performance, in '1985 Joint Power Generation Conference: GT Papers', American Society of Mechanical Engineers, pp. V001T03A001-V001T03A001.

White, A. J. (1992), Condensation in steam turbine cascades., PhD thesis, University of Cambridge.

White, A. \& Young, J. (1993), 'Time-marching method for the prediction of two-dimensional, unsteadyflows of condensing steam', Journal of Propulsion and Power 9(4), 579-587.

Wood, N. B. (1973), Flow unsteadiness and turbulence measurements in the low-pressure cylinder of a $500 \mathrm{MW}$ steam turbine, in 'Proceedings of IMechE Conference, London', Vol. 3, Mechanical Engineering Publications Bury St Edmunds and London.
Young, J. B. (1982), 'The Spontaneous Condensation of Steam in Supersonic nozzles', Physico Chemical hydrodynamics 3, 5782.

Young, J. B. (1992), 'Two-dimensional, nonequilibrium, wetsteam calculations for nozzles and turbine cascades', ASME J. Turbomach 114(3), 569-579.

Young, J. \& Yau, K. (1988), 'The inertial deposition of fog droplets on steam turbine blades', Journal of turbomachinery 110(2), 155-162.

Young, J., Yau, K. \& Walters, P. (1988), 'Fog droplet deposition and coarse water formation in low-pressure steam turbines: a combined experimental and theoretical analysis', Journal of Turbomachinery 110(2), 163-172. 